\title{
Supporting Information for \\ Oxidative Addition of the Chloromethane C-Cl Bond to Pd. An ab Initio Benchmark and DFT Validation Study
}

\author{
G. Theodoor de Jong and F. Matthias Bickelhaupt*
}

Afdeling Theoretische Chemie, Scheikundig Laboratorium der Vrije Universiteit, De Boelelaan 1083, NL-1081 HV Amsterdam, The Netherlands

Fax: +31 - 20 - 59 87629. E-mail: fm.bickelhaupt@few.vu.nl.

Table S1. Selected geometry parameters (in $\AA$, degrees), optimized with eight different density functionals and the TZ2P basis set with frozen-core approximation, of the stationary points along the reaction coordinates of the OxIn and $\mathrm{S}_{\mathrm{N}}$ 2-type pathways for oxidative addition of the $\mathrm{C}-\mathrm{Cl}$ bond of $\mathrm{CH}_{3} \mathrm{Cl}$ to $\mathrm{Pd}$.

Table S2. Total energies (in a.u.) of species occurring at stationary points along the reaction coordinate of the two pathways $\left(\mathrm{OxIn}\right.$ and $\mathrm{S}_{\mathrm{N}}$ ) for oxidative addition of the chloromethane $\mathrm{C}-\mathrm{Cl}$ bond to $\mathrm{Pd}$.

Table S3. Basis set superposition error (BSSE, in $\mathrm{kcal} / \mathrm{mol}$ ) for $\mathrm{Pd}$ and $\mathrm{CH}_{3} \mathrm{Cl}$ in the stationary points along the reaction coordinates of the OxIn and $\mathrm{S}_{\mathrm{N}}$ 2-type pathways for oxidative addition of the $\mathrm{C}-\mathrm{Cl}$ bond of $\mathrm{CH}_{3} \mathrm{Cl}$ to $\mathrm{Pd}$, computed at several levels of ab initio theory.

Table S4. Total energies (in a.u.) of the reactants and $\mathrm{S}_{\mathrm{N}} 2$-ra transition state for oxidative addition of the chloromethane $\mathrm{C}-\mathrm{Cl}$ bond to $\mathrm{PdCl}^{-}$.

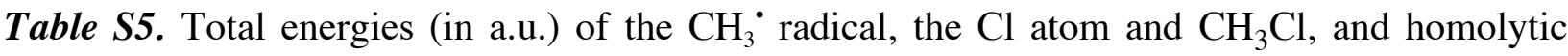
dissociation energy $D_{\mathrm{CCl}}$ (in $\mathrm{kcal} / \mathrm{mol}$ ) of the chloromethane $\mathrm{C}-\mathrm{Cl}$ bond

Figure S1. Structures of stationary points along the reaction coordinates of the OxIn and $\mathrm{S}_{\mathrm{N}} 2$ type pathways for oxidative addition of the $\mathrm{C}-\mathrm{Cl}$ bond of $\mathrm{CH}_{3} \mathrm{Cl}$ to $\mathrm{Pd}$. See Table $\mathrm{S} 1$ for values of geometry parameters.

Figure S2. Reaction profiles for the OxIn and $\mathrm{S}_{\mathrm{N}}$ 2-type pathways for oxidative addition of Pd to the $\mathrm{C}-\mathrm{Cl}$ bond of $\mathrm{CH}_{3} \mathrm{Cl}$, computed with $\mathrm{CCSD}(\mathrm{T})$ for various basis sets, without (upper panel) and with counterpoise correction (lower panel). Geometries optimized at ZORA-BLYP/TZ2P, i.e., with frozen-core approximation.

Figure S3. Reaction profiles for the two reaction pathways for the oxidative addition of Pd to the $\mathrm{C}-\mathrm{Cl}$ bond of $\mathrm{CH}_{3} \mathrm{Cl}$, obtained with seven different GGA density functionals (thin lines) and the TZ2P basis set with frozen-core approximation (geometries and energies computed at the same level of DFT; relativistic effects are treated with ZORA). The counterpoise-corrected CCSD(T) benchmark of this work is also included (thick lines).

Figure S4. Reaction profiles for the OxIn and $\mathrm{S}_{\mathrm{N}} 2$-type reaction pathways for oxidative addition of $\mathrm{Pd}$ to the $\mathrm{C}-\mathrm{Cl}$ bond of $\mathrm{CH}_{3} \mathrm{Cl}$, computed with ZORA-BLYP and four different basis sets with all electrons treated variationally, without counterpoise correction. Geometries optimized at ZORA-BLYP/TZ2P, i.e., with frozen-core approximation. 
Table S1. Selected geometry parameters a (in $\AA$, degrees), optimized with eight different density functionals and the TZ2P basis set with frozen-core approximation, ${ }^{\mathrm{b}}$ of the stationary points along the reaction coordinates of the OxIn and $\mathrm{S}_{\mathrm{N}} 2$-type pathways for oxidative addition of the $\mathrm{C}-\mathrm{Cl}$ bond of $\mathrm{CH}_{3} \mathrm{Cl}$ to $\mathrm{Pd}$.

\begin{tabular}{|c|c|c|c|c|c|c|c|c|c|}
\hline Method & & $\mathrm{C}-\mathrm{Cl}$ & $\mathrm{C}-\mathrm{H}(1)$ & $\mathrm{C}-\mathrm{H}(2)$ & $\mathrm{Pd}-\mathrm{C}$ & $\mathrm{Pd}-\mathrm{Cl}$ & $\mathrm{Pd}-\mathrm{H}$ & $\angle(\mathrm{Cl}-\mathrm{C}-\mathrm{H}(1))$ & $\angle(\mathrm{C}-\mathrm{Pd}-\mathrm{Cl})$ \\
\hline \multirow[t]{6}{*}{ VWN } & $\mathrm{R}$ & 1.771 & 1.097 & & & & & 108.8 & \\
\hline & $\mathrm{RC}_{\text {OxIn }}$ & 1.800 & 1.098 & 1.095 & 3.316 & 2.188 & & 107.0 & 30.2 \\
\hline & $\mathrm{RC}_{\mathrm{S}_{\mathrm{N}} 2}$ & 1.777 & 1.155 & 1.096 & 2.129 & 3.417 & 1.844 & 108.5 & 26.2 \\
\hline & $\mathrm{TS}_{\text {OxIn }}$ & 1.860 & 1.094 & 1.123 & 2.462 & 2.286 & 1.992 & 104.9 & 45.9 \\
\hline & $\mathrm{TS}_{\mathrm{S}_{\mathrm{N} 2}-\mathrm{ra}}$ & 2.984 & 1.194 & 1.102 & 1.935 & 3.472 & 2.123 & 5.3 & 59.1 \\
\hline & $\mathrm{P}^{\mathrm{c}}$ & 3.006 & 1.098 & 1.104 & 1.939 & 2.169 & 2.439 & 67.0 & 93.9 \\
\hline \multirow[t]{6}{*}{ BP86 } & $\mathrm{R}$ & 1.803 & 1.093 & & & & & 108.2 & \\
\hline & $\mathrm{RC}_{\text {OxIn }}$ & 1.836 & 1.094 & 1.092 & 3.451 & 2.275 & & 106.6 & 29.1 \\
\hline & $\mathrm{RC}_{\mathrm{S}_{\mathrm{N}} 2}$ & 1.808 & 1.131 & 1.092 & 2.279 & 3.627 & 1.944 & 107.7 & 24.2 \\
\hline & $\mathrm{TS}_{\text {OxIn }}$ & 1.963 & 1.089 & 1.118 & 2.432 & 2.355 & 2.020 & 101.5 & 48.4 \\
\hline & $\mathrm{TS}_{\mathrm{S}_{\mathrm{N}} 2-\mathrm{ra}}$ & 3.112 & 1.141 & 1.098 & 1.993 & 3.841 & 2.289 & 5.5 & 53.8 \\
\hline & $\mathrm{P}$ & 3.133 & 1.095 & 1.105 & 1.981 & 2.222 & 2.427 & 85.8 & 96.2 \\
\hline \multirow[t]{6}{*}{ BLYP } & $\mathrm{R}$ & 1.826 & 1.091 & & & & & 107.9 & \\
\hline & $\mathrm{RC}_{\text {OxIn }}$ & 1.862 & 1.091 & 1.089 & 3.526 & 2.335 & & 106.3 & 28.9 \\
\hline & $\mathrm{RC}_{\mathrm{S}_{\mathrm{N}} 2}$ & 1.831 & 1.117 & 1.089 & 2.398 & 3.785 & 2.037 & 107.4 & 22.9 \\
\hline & $\mathrm{TS}_{\text {OxIn }}$ & 2.054 & 1.085 & 1.102 & 2.521 & 2.365 & 2.154 & 99.0 & 49.6 \\
\hline & $\mathrm{TS}_{\mathrm{S}_{\mathrm{N}} 2 \text {-ra }}$ & 3.202 & 1.121 & 1.095 & 2.025 & 4.046 & 2.360 & 6.3 & 51.4 \\
\hline & $\mathrm{P}$ & 3.221 & 1.092 & 1.103 & 2.007 & 2.256 & 2.449 & 86.0 & 98.0 \\
\hline \multirow[t]{6}{*}{ PW91 } & $\mathrm{R}$ & 1.798 & 1.091 & & & & & 108.3 & \\
\hline & $\mathrm{RC}_{\text {OxIn }}$ & 1.830 & 1.092 & 1.090 & 3.450 & 2.275 & & 106.7 & 29.0 \\
\hline & $\mathrm{RC}_{\mathrm{S}_{\mathrm{N}} 2}$ & 1.804 & 1.129 & 1.090 & 2.277 & 3.622 & 1.942 & 107.7 & 24.1 \\
\hline & $\mathrm{TS}_{\text {OxIn }}$ & 1.948 & 1.088 & 1.118 & 2.423 & 2.362 & 2.002 & 101.9 & 48.0 \\
\hline & $\mathrm{TS}_{\mathrm{S}_{\mathrm{N}} 2-\mathrm{ra}}$ & 3.100 & 1.140 & 1.097 & 1.991 & 3.837 & 2.287 & 5.8 & 53.6 \\
\hline & $\mathrm{Pc}$ & 3.113 & 1.092 & 1.099 & 1.980 & 2.221 & 2.475 & 67.1 & 95.5 \\
\hline \multirow[t]{6}{*}{ PBE } & $\mathrm{R}$ & 1.797 & 1.094 & & & & & 108.3 & \\
\hline & $\mathrm{RC}_{\text {OxIn }}$ & 1.828 & 1.094 & 1.092 & 3.445 & 2.273 & & 106.7 & 29.0 \\
\hline & $\mathrm{RC}_{\mathrm{S}_{\mathrm{N}} 2}$ & 1.803 & 1.132 & 1.092 & 2.276 & 3.618 & 1.942 & 107.8 & 24.2 \\
\hline & $\mathrm{TS}_{\text {OxIn }}$ & 1.944 & 1.090 & 1.122 & 2.413 & 2.366 & 1.991 & 102.0 & 48.0 \\
\hline & $\mathrm{TS}_{\mathrm{S}_{\mathrm{N}} 2-\mathrm{ra}}$ & 3.106 & 1.142 & 1.099 & 1.991 & 3.844 & 2.292 & 5.6 & 53.6 \\
\hline & $\mathrm{P}$ & 3.113 & 1.095 & 1.106 & 1.978 & 2.221 & 2.425 & 85.5 & 95.5 \\
\hline \multirow[t]{6}{*}{ revPBE } & $\mathrm{R}$ & 1.805 & 1.094 & & & & & 108.3 & \\
\hline & $\mathrm{RC}_{\text {OxIn }}$ & 1.837 & 1.095 & 1.093 & 3.501 & 2.305 & & 106.6 & 28.4 \\
\hline & $\mathrm{RC}_{\mathrm{S}_{\mathrm{N}} 2}$ & 1.811 & 1.126 & 1.093 & 2.347 & 3.719 & 1.995 & 107.6 & 23.1 \\
\hline & $\mathrm{TS}_{\text {OxIn }}$ & 1.977 & 1.090 & 1.120 & 2.424 & 2.380 & 2.025 & 101.0 & 48.6 \\
\hline & $\mathrm{TS}_{\mathrm{S}_{\mathrm{N}} 2 \text {-ra }}$ & 3.172 & 1.131 & 1.099 & 2.006 & 3.981 & 2.347 & 5.4 & 52.1 \\
\hline & $\mathrm{P}$ & 3.150 & 1.096 & 1.106 & 1.989 & 2.236 & 2.438 & 85.7 & 96.2 \\
\hline \multirow[t]{6}{*}{ RPBE } & $\mathrm{R}$ & 1.807 & 1.094 & & & & & 108.2 & \\
\hline & $\mathrm{RC}_{\text {OxIn }}$ & 1.839 & 1.095 & 1.093 & 3.512 & 2.316 & & 106.7 & 28.4 \\
\hline & $\mathrm{RC}_{\mathrm{S}_{\mathrm{N}} 2}$ & 1.831 & 1.124 & 1.093 & 2.371 & 3.748 & 2.014 & 107.6 & 22.8 \\
\hline & $\mathrm{TS}_{\text {OxIn }}$ & 1.987 & 1.090 & 1.119 & 2.429 & 2.384 & 2.037 & 100.7 & 48.7 \\
\hline & $\mathrm{TS}_{\mathrm{S}_{\mathrm{N}} 2-\mathrm{ra}}$ & 3.187 & 1.129 & 1.100 & 2.010 & 4.016 & 2.360 & 5.5 & 51.7 \\
\hline & $\mathrm{P}$ & 3.158 & 1.096 & 1.106 & 1.992 & 2.241 & 2.441 & 85.7 & 96.3 \\
\hline \multirow[t]{6}{*}{ OLYP } & $\mathrm{R}$ & 1.792 & 1.090 & & & & & 108.4 & \\
\hline & $\mathrm{RC}_{\text {OxIn }}$ & 1.820 & 1.091 & 1.089 & 3.539 & 2.324 & & 106.9 & 27.3 \\
\hline & $\mathrm{RC}_{\mathrm{S}_{\mathrm{N}} 2}$ & 1.797 & 1.114 & 1.089 & 2.412 & 3.790 & 2.042 & 107.9 & 22.0 \\
\hline & $\mathrm{TS}_{\text {OxIn }}$ & 1.968 & 1.086 & 1.121 & 2.356 & 2.390 & 1.976 & 100.7 & 49.0 \\
\hline & $\mathrm{TS}_{\mathrm{S}_{\mathrm{N}} 2-\mathrm{ra}}$ & 3.227 & 1.117 & 1.095 & 1.996 & 4.093 & 2.377 & 5.1 & 50.8 \\
\hline & $\mathrm{P}$ & 3.146 & 1.091 & 1.102 & 1.974 & 2.230 & 2.419 & 86.0 & 96.7 \\
\hline
\end{tabular}

\footnotetext{
a See Figure S1 for definition.

b Relativistic effects treated with ZORA.

c $\mathrm{Pd}-\mathrm{Cl}$ bond orientated eclipsed with respect to $\mathrm{C}-\mathrm{H}(1)$ bond, at variance with the product geometries for the other functionals.
} 
Table S2. Total energies (in a.u.) of species occurring at stationary points along the reaction coordinate of the two pathways $\left(\mathrm{OxIn}\right.$ and $\mathrm{S}_{\mathrm{N}}$ ) for oxidative addition of the chloromethane $\mathrm{C}-\mathrm{Cl}$ bond to Pd.

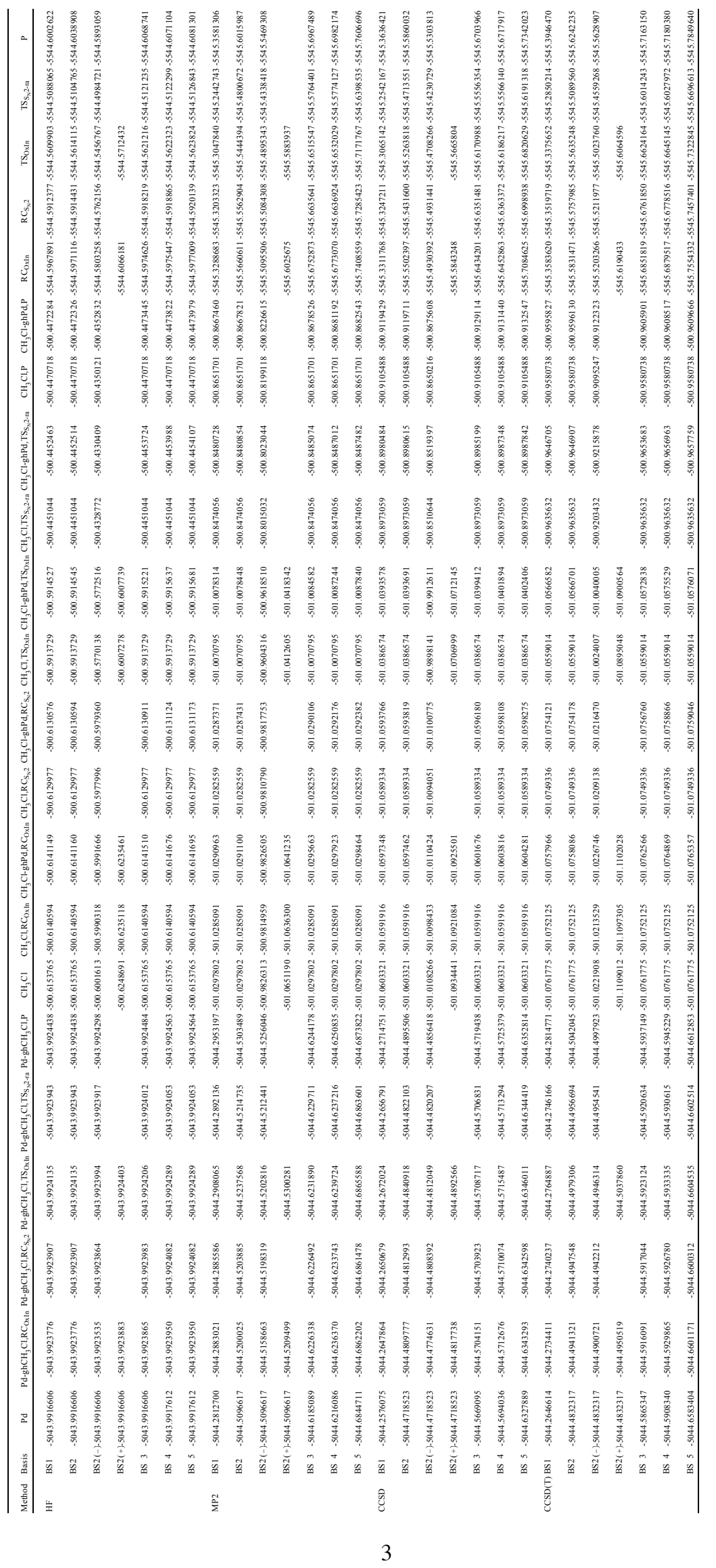


Table S3. Basis set superposition error (BSSE, in $\mathrm{kcal} / \mathrm{mol}$ ) for $\mathrm{Pd}$ and $\mathrm{CH}_{3} \mathrm{Cl}$ in the stationary points along the reaction coordinates of the OxIn and $\mathrm{S}_{\mathrm{N}} 2$-type pathways for oxidative addition of the $\mathrm{C}-\mathrm{Cl}$ bond of $\mathrm{CH}_{3} \mathrm{Cl}$ to $\mathrm{Pd}$, computed at several levels of ab initio theory.

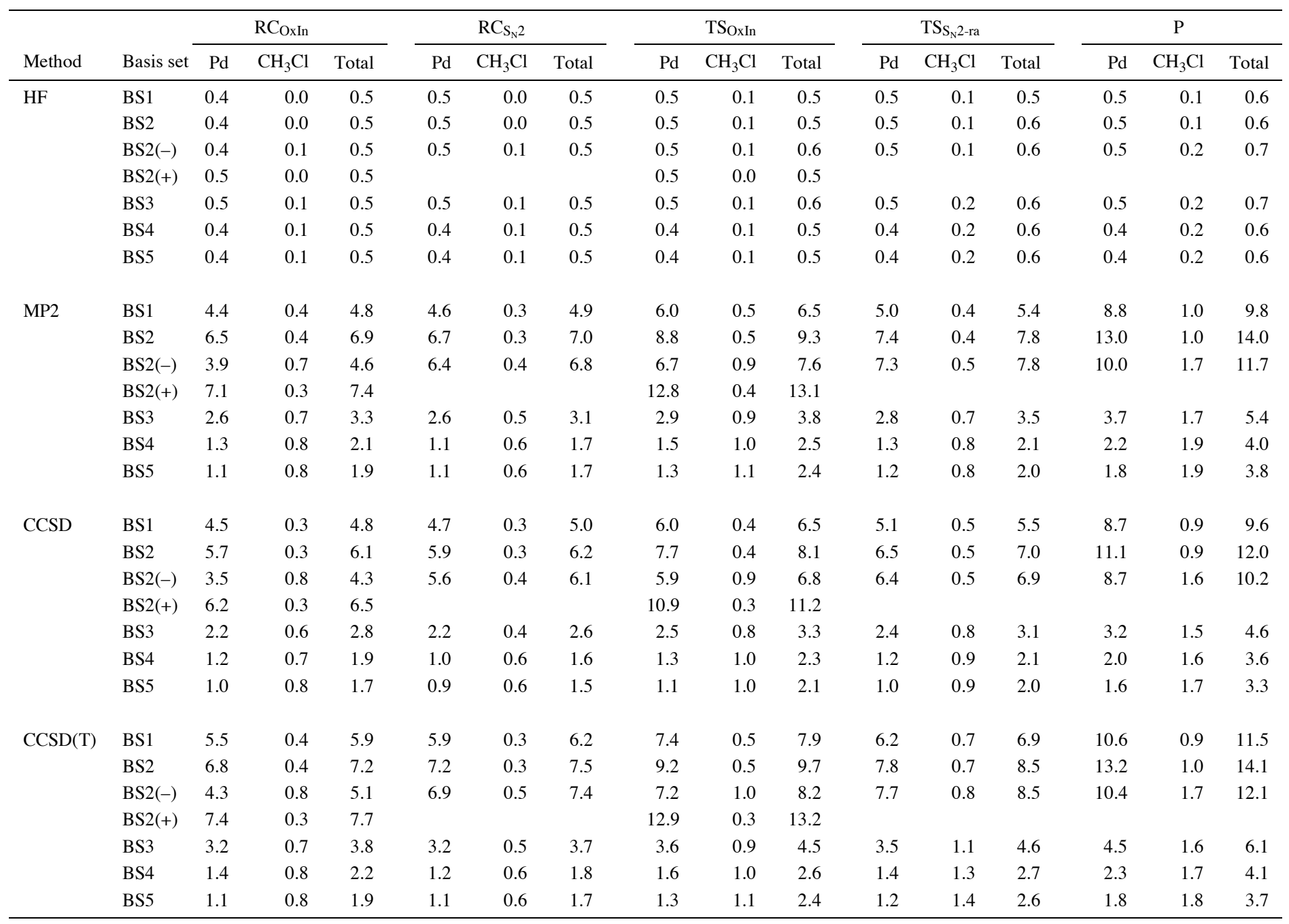


Table S4. Total energies ${ }^{\text {a }}$ (in a.u.) of the reactants and $\mathrm{S}_{\mathrm{N}} 2$-ra transition state for oxidative addition of the chloromethane $\mathrm{C}-\mathrm{Cl}$ bond to $\mathrm{PdCl}^{-}$.

\begin{tabular}{|c|c|c|c|c|c|c|c|}
\hline Method & $\mathrm{PdCl}^{-}$ & $\mathrm{PdCl}^{-}, \mathrm{TS}$ & $\mathrm{PdCl}^{-}-\mathrm{ghCH}_{3} \mathrm{Cl}, \mathrm{TS}$ & $\mathrm{CH}_{3} \mathrm{Cl}$ & $\mathrm{CH}_{3} \mathrm{Cl}, \mathrm{TS}$ & $\mathrm{CH}_{3} \mathrm{Cl}-\mathrm{ghPdCl}^{-}, \mathrm{TS}$ & $\mathrm{TS}$ \\
\hline $\mathrm{HF}$ & -5505.0160697 & -5505.0208531 & -5505.0219929 & -500.6153765 & -500.4347855 & -500.4350879 & -6005.6491387 \\
\hline MP2 & -5505.9159420 & -5505.9231973 & -5505.9314798 & -501.0297802 & -500.8390894 & -500.8403765 & -6006.9773369 \\
\hline CCSD & -5505.8623387 & -5505.8685988 & -5505.8761766 & -501.0603321 & -500.8922033 & -500.8936297 & -6006.9598762 \\
\hline
\end{tabular}

a Calculated with BS3 basis, with completely uncontracted cc-aug-pVTZ and cc-aug-pVDZ basis for substrate chlorine and palladium-ligating chlorine, respectively.

${ }^{\mathrm{b}}$ Geometries optimized at ZORA-BLYP/TZ2P. 
Table S5. Total energies a (in a.u.) of the $\mathrm{CH}_{3}{ }^{\cdot}$ radical, the $\mathrm{Cl}$ atom and $\mathrm{CH}_{3} \mathrm{Cl}$, and homolytic dissociation energy $D_{\mathrm{CCl}}$ (in $\mathrm{kcal} / \mathrm{mol}$ ) of the chloromethane $\mathrm{C}-\mathrm{Cl}$ bond. ${ }^{\mathrm{b}}$

\begin{tabular}{|c|c|c|c|c|c|c|c|}
\hline Method & $\mathrm{CH}_{3}^{\cdot}$ & $\mathrm{Cl}$ & $\mathrm{CH}_{3} \mathrm{Cl}$ & $\mathrm{CH}_{3}, \mathrm{CH}_{3} \mathrm{Cl}$ & $\mathrm{CH}_{3}{ }^{-}-\mathrm{ghCl}^{-} \mathrm{CH}_{3} \mathrm{Cl}$ & $\mathrm{Cl}$-ghCH${ }_{3}, \mathrm{CH}_{3} \mathrm{Cl}$ & $D_{\mathrm{CCl}}(\mathrm{kcal} / \mathrm{mol})$ \\
\hline $\mathrm{HF}$ & -39.5799094 & -460.9333775 & -500.6153765 & -39.5730405 & -39.5733994 & -460.9395098 & 59.99 \\
\hline MP2 & -39.7233071 & -461.1604338 & -501.0297802 & -39.7143147 & -39.7161347 & -461.1676465 & 85.97 \\
\hline CCSD & -39.7453640 & -461.1811086 & -501.0603321 & -39.7369327 & -39.7387659 & -461.1885228 & 78.20 \\
\hline $\operatorname{CCSD}(\mathrm{T})$ & -39.7492849 & -461.1881110 & -501.0761775 & -39.7408726 & -39.7428345 & -461.1955963 & 81.16 \\
\hline
\end{tabular}

a Calculated with completely uncontracted cc-aug-pVDZ basis for $\mathrm{C}$ and $\mathrm{H}$, and with completely uncontracted cc-aug-pVTZ basis for $\mathrm{Cl}$.

${ }^{b}$ Geometries optimized at ZORA-BLYP/TZ2P. 
Figure S1. Structures of stationary points along the reaction coordinates of the OxIn and $\mathrm{S}_{\mathrm{N}} 2$ type pathways for oxidative addition of the $\mathrm{C}-\mathrm{Cl}$ bond of $\mathrm{CH}_{3} \mathrm{Cl}$ to $\mathrm{Pd}$. See Table $\mathrm{S} 1$ for values of geometry parameters.
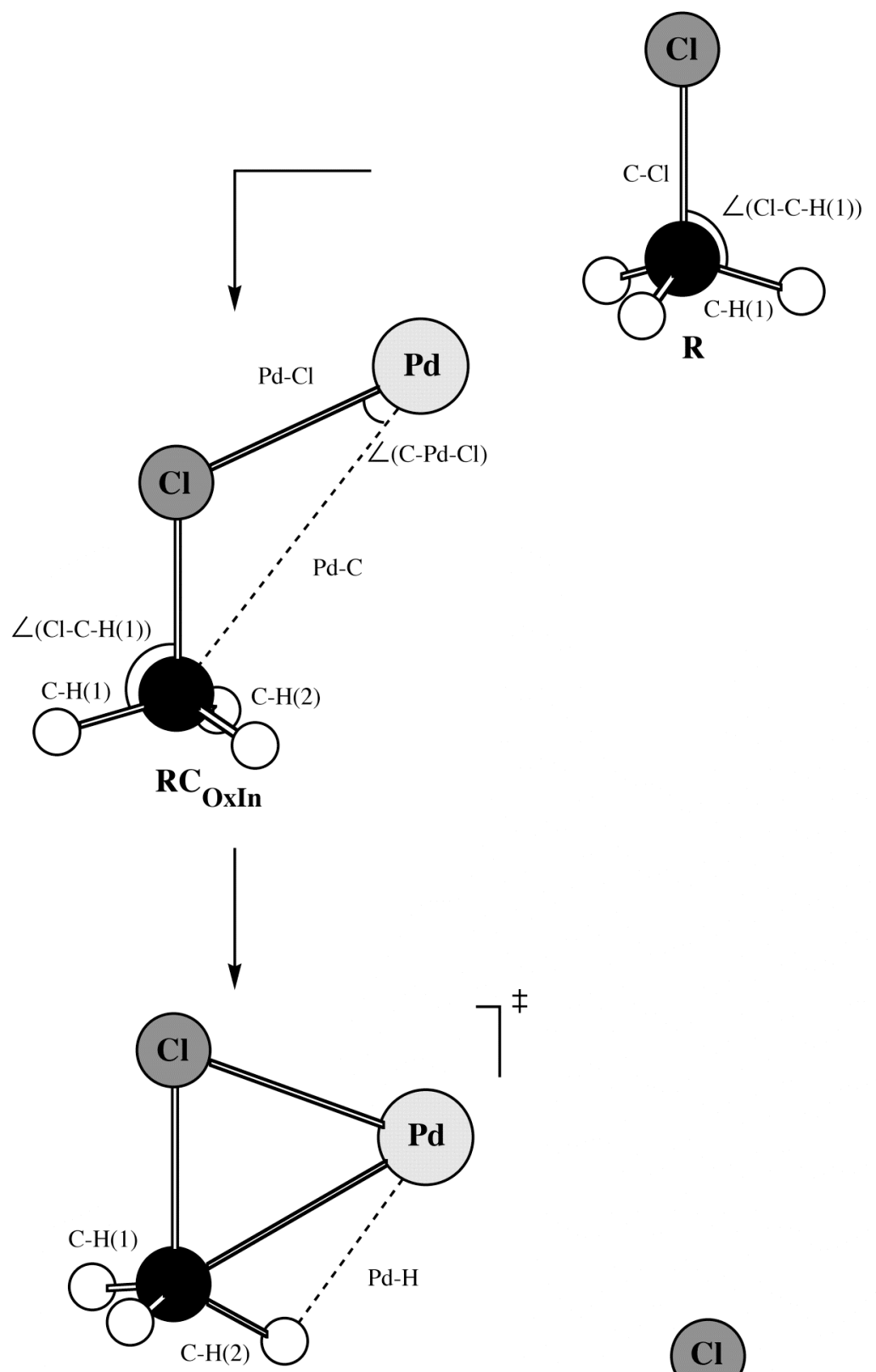

TS OxIn
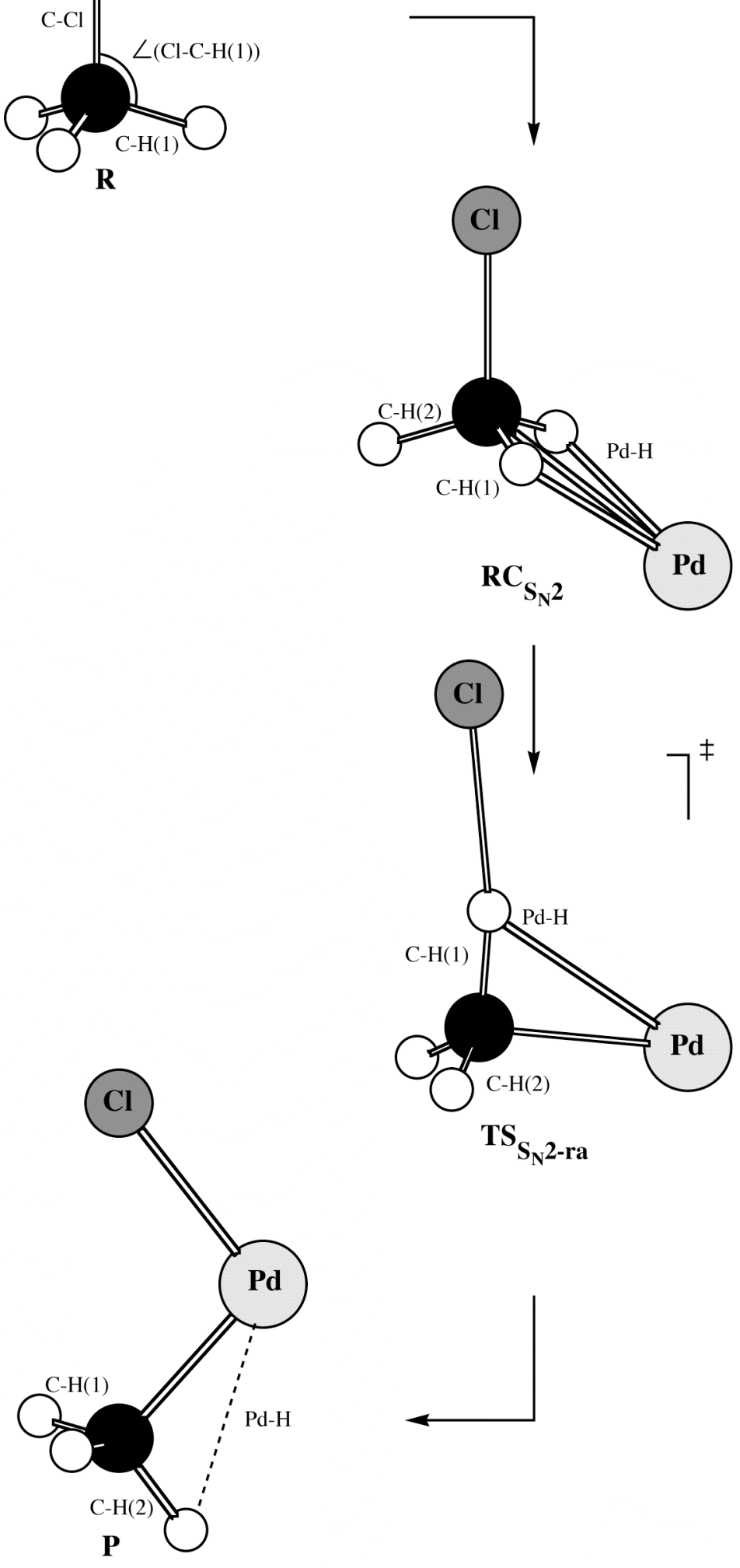
Figure S2. Reaction profiles for the OxIn and $\mathrm{S}_{\mathrm{N}}$ 2-type pathways for oxidative addition of $\mathrm{Pd}$ to the $\mathrm{C}-\mathrm{Cl}$ bond of $\mathrm{CH}_{3} \mathrm{Cl}$, computed with $\mathrm{CCSD}(\mathrm{T})$ for various basis sets, without (upper panel) and with counterpoise correction (lower panel). Geometries optimized at ZORA-BLYP/TZ2P, i.e., with frozen-core approximation.

$\operatorname{CCSD}(\mathrm{T})$

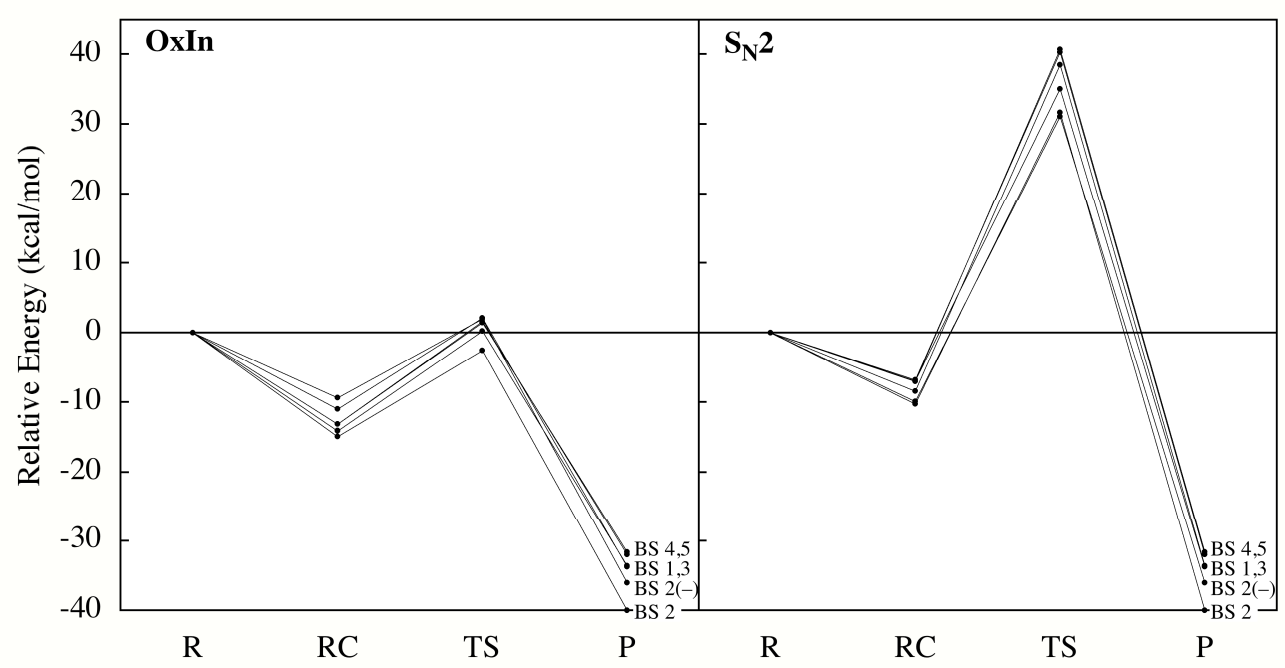

CCSD(T) with CPC

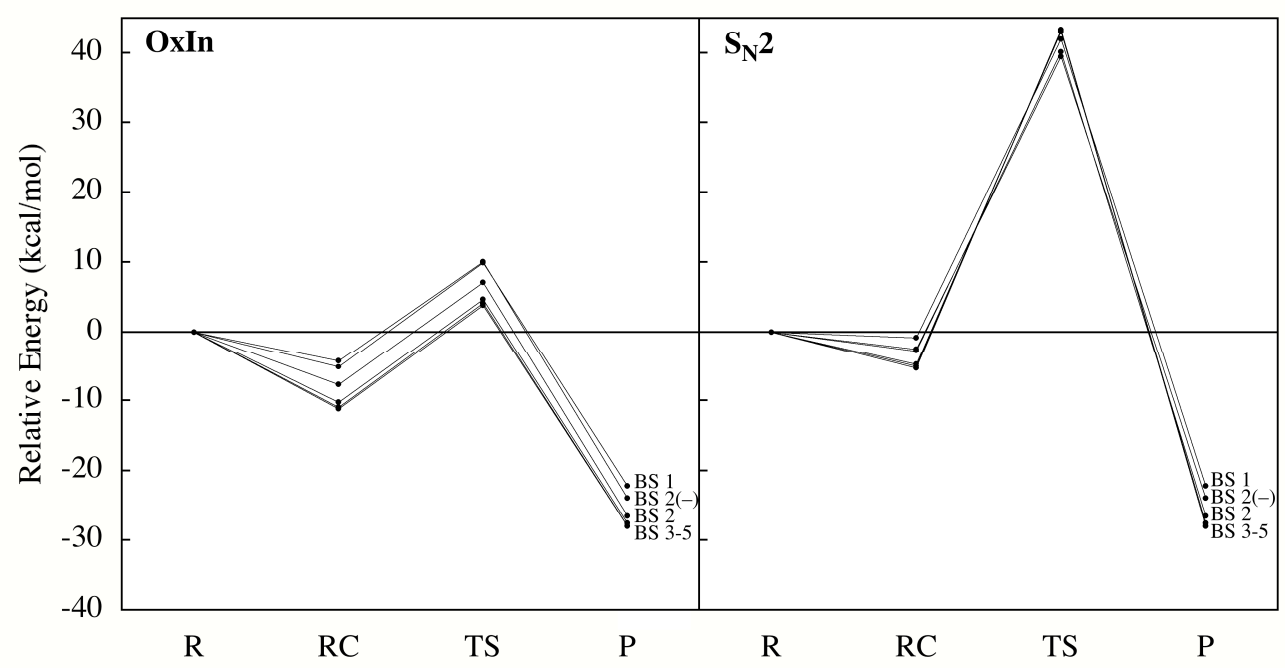


Figure S3. Reaction profiles for the two reaction pathways for the oxidative addition of Pd to the $\mathrm{C}-\mathrm{Cl}$ bond of $\mathrm{CH}_{3} \mathrm{Cl}$, obtained with seven different GGA density functionals (thin lines) and the TZ2P basis set with frozen-core approximation (geometries and energies computed at the same level of DFT; relativistic effects are treated with ZORA). The counterpoise-corrected CCSD(T) benchmark of this work is also included (thick lines).

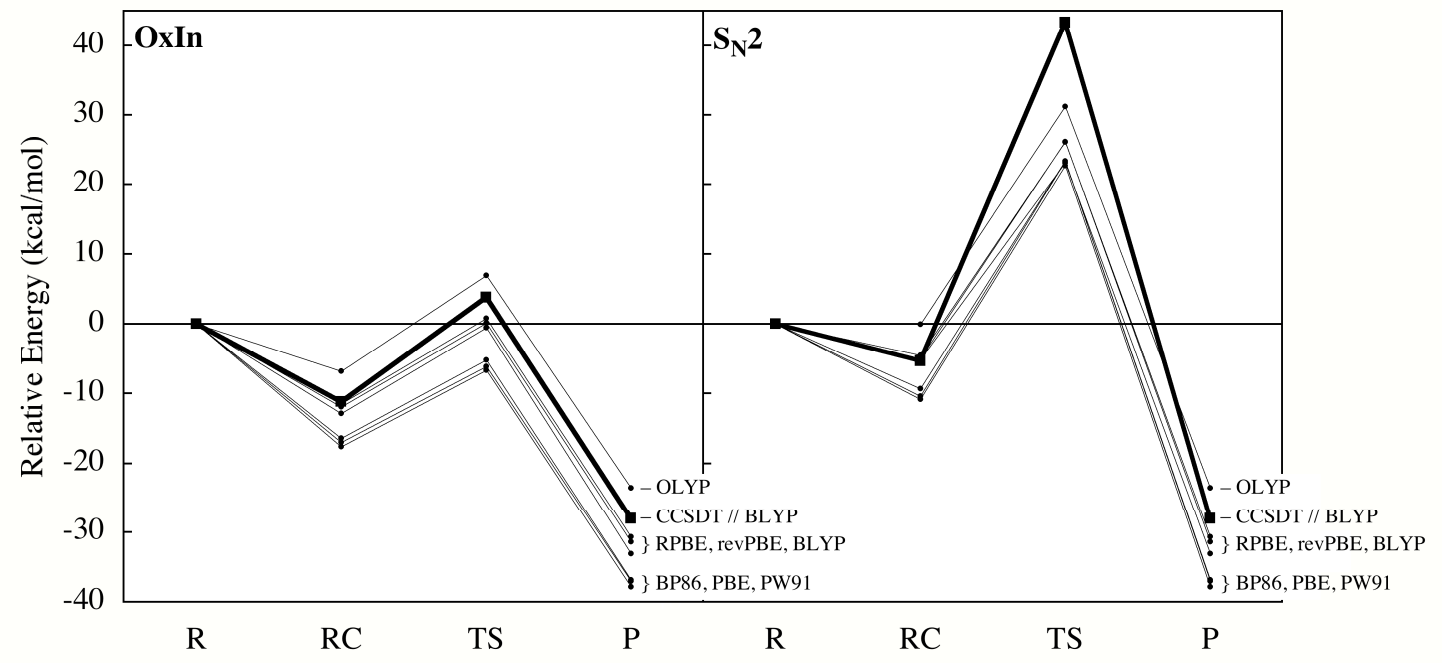


Figure S4. Reaction profiles for the OxIn and $\mathrm{S}_{\mathrm{N}}$ 2-type reaction pathways for oxidative addition of $\mathrm{Pd}$ to the $\mathrm{C}-\mathrm{Cl}$ bond of $\mathrm{CH}_{3} \mathrm{Cl}$, computed with ZORA-BLYP and four different basis sets with all electrons treated variationally, without counterpoise correction. Geometries optimized at ZORA-BLYP/TZ2P, i.e., with frozen-core approximation.

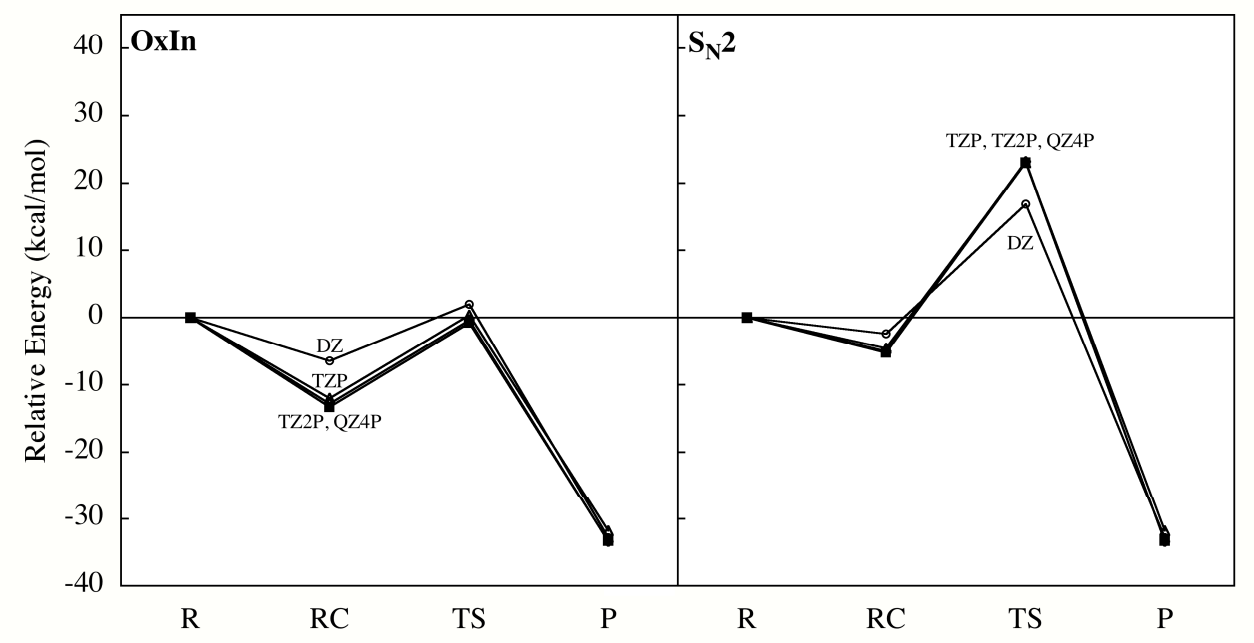

